\title{
Imaging HII Regions from Galaxies and Quasars During Reionisation with SKA
}

\author{
J. Stuart B. Wyithe*, Paul M. Geil, Hansik Kim \\ School of Physics, The University of Melbourne, Parkville, Vic 3010, Australia \\ E-mail: swyithe@unimelb.edu.au
}

\begin{abstract}
The ionisation structure of the Intergalactic Medium (IGM) during reionisation is sensitive to the unknown galaxy formation physics that prevailed at that time. This structure introduces non-Gaussian statistics into the redshifted $21 \mathrm{~cm}$ fluctuation amplitudes that can only be studied through tomographic imaging, which will clearly discriminate between different galaxy formation scenarios. Imaging the ionisation structure and cosmological HII regions during reionisation is therefore a key goal for the SKA. For example, the SKA1-LOW baseline design with a $1 \mathrm{~km}$ diameter core will resolve HII regions expected from galaxy formation models which include strong feedback on low-mass galaxy formation. Imaging the smaller HII regions that result from galaxy formation in the absence of SNe feedback will also be possible for SKA1-LOW in the later stages of reionisation, but may require the greater sensitivity of SKA early in the reionisation era. In addition to having baselines long enough to resolve the HII regions, the field of view for SKA1-LOW reionisation experiments should be at least several degrees in order to image the largest HI structures towards the end of reionisation. The baseline design with 35 meter diameter stations has a field of view within a single primary pointing which is sufficient for this purpose.
\end{abstract}

Advancing Astrophysics with the Square Kilometre Array

June 8-13, 2014

Giardini Naxos, Italy

\footnotetext{
* Speaker.
} 


\section{Introduction}

Redshifted $21 \mathrm{~cm}$ emission from HI in the intergalactic medium (IGM) offers several probes of the reionisation epoch. Owing to the low signal-to-noise expected for first generation telescopes, including the MWA and LOFAR, most attention has been focussed on statistical observations of the power-spectrum of $21 \mathrm{~cm}$ fluctuations together with its evolution. However several other observational signatures become more powerful probes of reionisation using the imaging capability of SKA1-LOW. These include $i$ ) the cross-correlation of $21 \mathrm{~cm}$ emission with galaxies, which would directly probe the connection between reionisation and the sources of ionising radiation (Wyithe \& Loeb, 2007b; Furlanetto \& Lidz, 2007; Lidz et al., 2009; Park et al., 2014), ii) the probability distribution of intensity fluctuations, which contains additional information about the non-Gaussian intensity fluctuations during reionisation (Wyithe \& Loeb, 2007b; Harker et al., 2009a; Barkana, 2009), iii) the observation of individual HII regions, which will probe galaxy formation physics, and $i v$ ) the observation of quasar-dominated HII regions, which will probe quasar emission geometries as well as the evolution of the neutral IGM (Wyithe \& Loeb, 2004a; Kohler et al., 2005; Valdés et al., 2006).

While the cross-correlation of $21 \mathrm{~cm}$ emission with galaxies may be possible with first generation arrays using the statistics of the cross power-spectrum (Wyithe \& Loeb, 2007b; Furlanetto \& Lidz, 2007; Lidz et al., 2009), direct observation of the correspondence between galaxies and ionisation structure will provide unambiguous evidence of the role of galaxies in reionisation. An important prediction from the various modelling studies concerns whether over-dense or under-dense regions become ionised first. Standard calculations of the expected cross-correlation between the distribution of galaxies and the intergalactic $21 \mathrm{~cm}$ emission at high redshifts show that over-dense regions will be ionised early (e.g. Wyithe \& Loeb, 2007b), leading to an anti-correlation between $21 \mathrm{~cm}$ emission and the galaxy population. Redshifted $21 \mathrm{~cm}$ surveys should be able to discriminate between "outside-in" and "inside-out" reionisation, even when combined with galaxy surveys of only several square degrees.

The distribution of fluctuation amplitudes is expected to be Gaussian during the early phases of reionisation. At these early times the power-spectrum represents the natural quantity to describe fluctuations in $21 \mathrm{~cm}$ emission, since it contains all the statistical information. However the distribution of fluctuation amplitudes becomes non-Gaussian as reionisation progresses (Mellema et al., 2006; Wyithe \& Morales, 2007; Harker et al., 2009b). This is particularly the case on small scales once HII regions have formed (Furlanetto et al., 2004). As a result, the power-spectrum does not provide a complete statistical description of the reionisation process, and instead the full probability distribution of intensity fluctuations should be considered. For example, additional information could be captured by measuring skewness in the probability distribution (Wyithe \& Morales, 2007; Harker et al., 2009b). However, unlike the power-spectrum, the probability distribution for intensity fluctuations must be derived from images of the ionisation structure.

In this chapter we focus on the prospects for studying galaxy formation through the imaging of HII regions, both in the typical IGM as well as around quasars using SKA1-LOW. The direct imaging of the ionised structure during reionisation would be the most unambiguous measurement of reionisation. This represents the "holy grail" of $21 \mathrm{~cm}$ reionisation experiments, and is a key goal for the SKA. The importance of imaging with SKA for capturing the physics of reionisation 
is discussed in more detail in Mellema et al. (2015). Discussion of the science potential of SKA1LOW for imaging the ionised structure during reionisation requires modelling of ionised regions on very large scales, and we therefore begin this chapter with a brief discussion of the semi-numerical method used to calculate ionisation structure. A more detailed discussion of issues associated with simulation of reionisation can be found in Iliev et al. (2015).

\section{Semi-numerical model for reionisation}

In recent years approximate, but efficient semi-numerical methods for simulating the reionisation process have been developed (Mesinger \& Furlanetto, 2007) in order to overcome the limitations of both analytic and numerical methods. These extend prior work (Bond \& Myers, 1996; Zahn et al., 2007) to estimate the ionisation field based on a catalogue of sources by applying a filtering technique based on an analytic HII region model (Furlanetto et al., 2004). The resulting structure of the ionisation field is similar for different variations on the filtering method as well as for full radiative transfer, implying that semi-numerical models can be used to explore a larger range of reionisation scenarios than is possible with current numerical simulations (Mesinger \& Furlanetto, 2007). Alternatively, rather than adopt a filtering scheme, ionising sources can be used used to generate HII regions based on the spherically averaged radial density profile (Thomas et al., 2009). A feature of this model is that overlap of neighbouring HII regions is treated self-consistently with respect to photon conservation. The model can also be easily adapted to compute ionisation structure for more complex galaxy formation models.

We use a combination of these techniques implemented within the semi-analytic model GALFORM (e.g. Lagos et al., 2012) in order to study the effect of galaxy formation properties on the ionisation structure of the IGM (Kim et al., 2013). Beginning with a relatively small $(\sim 100$ $\mathrm{Mpc} / h$ cubed) volume simulation of the ionisation structure in which the physics of the lowest mass sources thought to dominate reionisation are resolved, this method can be extended to very large volumes using the statistical distribution of ionising flux as a function of large-scale $(\sim \mathrm{Mpc})$ over density (Kim 2014, in prep). This distribution is used to populate a much larger low resolution volume via a Monte-Carlo technique, thus producing simulations of ionisation structure in cubic Gpc volumes that contain the SKA1-LOW field of view, while retaining the effects of physics from the smallest-scale galaxies.

\subsection{HII regions and galaxy formation}

Examples of slices through simulated volumes of ionisation structure are shown in Figure 1. Several different volumes are shown at $z=7.272$ to illustrate the scales involved. All simulations have the same mass-averaged neutral hydrogen fraction of $\left\langle x_{\mathrm{HI}}\right\rangle \sim 0.45$. From left to right we show the HII region structure resulting from the default GALFORM galaxy formation model implemented within the $100 \mathrm{Mpc} / h$ cubed volume of the Millennium-II simulation (Kim et al., 2013), as well as the extension via our Monte-Carlo method (Kim 2014 in prep) to the $500 \mathrm{Mpc} / \mathrm{h}$ cubed volume of the Millennium simulation and $1 \mathrm{Gpc} / h$ cubed volume of the GiggleZ simulation (Poole et al., 2014). Note that ionised volumes that are nearly as large as the $100 \mathrm{Mpc} / h$ cubed Millennium-II simulation can be seen in the larger $500 \mathrm{Mpc} / h$ cubed Millennium and $1 \mathrm{Gpc} / h$ 


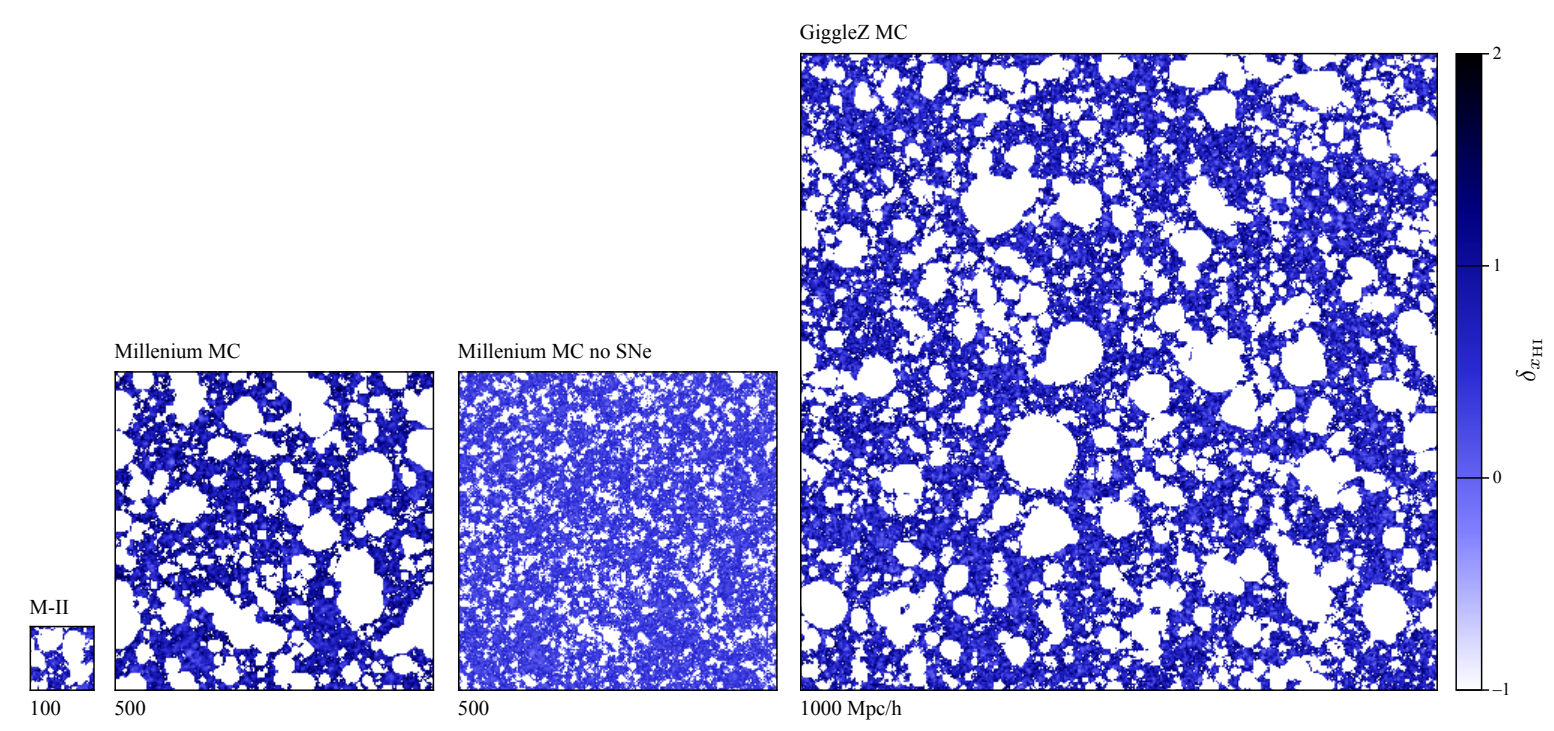

Figure 1: Simulations of the HII region structure in the Millennium-II simulation (left), with Monte-Carlo (MC) extension to the Millennium simulation (second and third panels) and the Gigglez 1Gpc cubed simulation (right panel). In each case simulations are shown for the default GALFORM galaxy formation model with SNe feedback, and reionisation was assumed to have progressed to a neutral fraction of 0.45 . In the case of the Millennium simulation we also show the scenario where the galaxy formation model does not include SNe feedback (third panel from left). The model slices have a depth $2 \mathrm{Mpc} / h$.

cubed GiggleZ simulation volumes. These very large HII regions indicate that Gpc-scale volumes are required to fully understand the large-scale power of reionisation (Iliev et al., 2014).

In addition to the default GALFORM galaxy formation model in which $\mathrm{SNe}$ feedback plays a significant role in regulating star formation, for the Millennium $500 \mathrm{Mpc} / h$ cubed simulation we also show a second example in which $\mathrm{SNe}$ feedback is absent. In each case the galaxy formation model produces an acceptable description of the high redshift galaxy luminosity function, and the mean neutral fraction of the IGM in both models is forced to be equal by construction. In the case of a galaxy formation model without $\mathrm{SNe}$ feedback, we find that much smaller HII regions are produced owing to the smaller luminosity-weighted mass of the ionising galaxies (Kim et al., 2013), requiring imaging at greater resolution in order to study the structure of the HII regions.

\subsection{Quasar HII regions}

Spectra of several of the most distant known quasars at $z \sim 6$ exhibit evidence for the presence of an HII region in a partially neutral IGM e.g. (Cen \& Haiman, 2000; Wyithe \& Loeb, 2004a), although this interpretation remains uncertain (Lidz et al., 2007; Bolton \& Haehnelt, 2007). Recently, stronger evidence for a neutral IGM has been found at $z \sim 7$ (Bolton et al., 2011), where spectra show the possible detection of an IGM-generated Ly $\alpha$ damping wing in addition to a Ly $\alpha$ absorption trough. The redshifted $21 \mathrm{~cm}$ observation of quasar HII regions will probe quasar physics as well as the evolution of the neutral gas (Wyithe \& Loeb, 2004a; Kohler et al., 2005). For example, Figure 2 shows a semi-numerical model of the evolving 3-dimensional ionisation structure of the IGM within a $500 \mathrm{Mpc}$ cubed volume around a luminous $z>6$ quasar (Geil et al., 2008) observed 

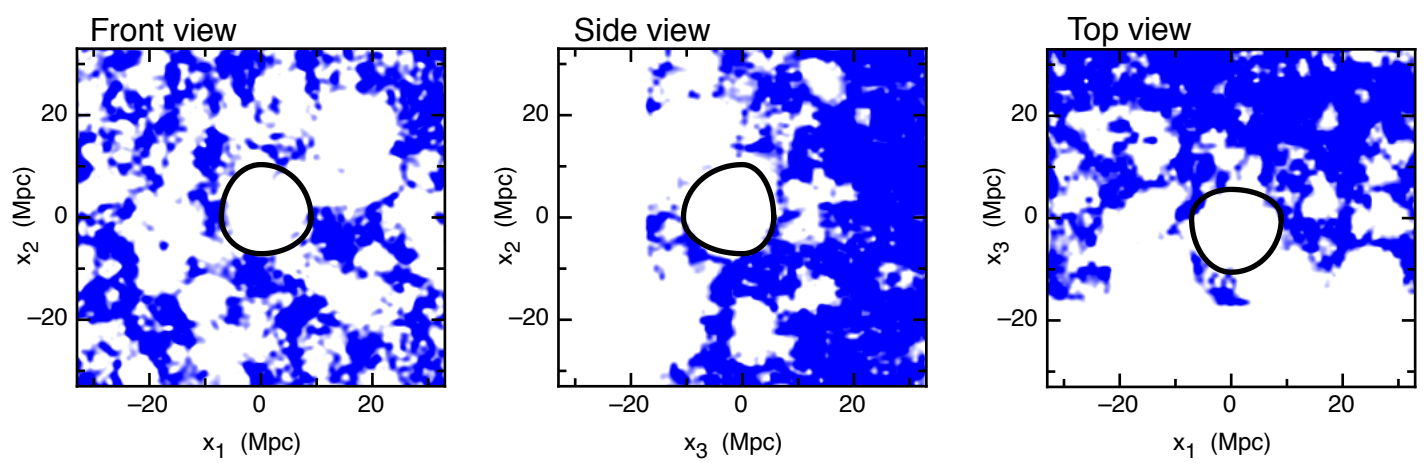

Figure 2: A quasar HII region in an evolving IGM (figure adapted from Geil et al., 2008). Three aspects of the HII region are shown, with slices through the centre of the box when viewed from the front, top and side. The quasar was assumed to contribute ionisation equivalent to an HII region of radius $R_{\mathrm{q}}=34$ co-moving $\mathrm{Mpc}$, and to be centred on $z=6.65$, which is also the redshift at the centre of the simulation box. Each slice is $6 \mathrm{Mpc}$ thick, which corresponds to $\sim 3 \mathrm{MHz}$ along the $x_{3}$-axis (units in this figure are shown in physical $\mathrm{Mpc}$ ). In observed units, the cube is $\sim 3.3$ degrees on a side and $33 \mathrm{MHz}$ deep. The shape of the HII region is also plotted (see Geil et al., 2008, for details). The mass-averaged IGM neutral fraction was assumed to be 0.15 at the quasar redshift.

near the end of reionisation. The evolution of the IGM, which is assumed to be quite rapid, is clearly seen in this figure with the percolation process completing between the "back" of the box and the "front" of the box. We note that in difference to galaxy HII regions which are driven by many sources over a long period of time, during the early phase quasar driven HII regions expand with a relativistic speed (Wyithe \& Loeb, 2004b). Consequently, their measured sizes along and transverse to the line-of-sight should have different observed values due to relativistic time delay. A combined measurement of these sizes could therefore be used to directly constrain the neutral fraction of the surrounding IGM as well as the quasar lifetime (Wyithe \& Loeb, 2004b). The figure also illustrates asymmetries perpendicular to the line of sight, which are a result of expansion of the spherical quasar driven HII region into an in homogeneously ionised IGM.

\section{Imaging sensitivity of SKA1-LOW}

In this section we estimate the sensitivity of the proposed SKA1-LOW baseline design with respect to imaging of cosmological HII regions. The baseline design for the SKA1-LOW will consist of 949 stations, of which approximately 400 are located within a uniform station density profile within a $500 \mathrm{~m}$ radius, with the remaining stations distributed in equal area per logarithm of radius in spiral arms beyond the core. Each station consists of 256 dual-polarisation antenna elements within a diameter of $35 \mathrm{~m}$. The system temperature at $v<200 \mathrm{MHz}$ is due to the combined sky and receiver temperatures, with a value $T_{\text {sys }}=T_{\text {sky }}+T_{\text {rcvr }}$, where $T_{\text {sky }} \sim 60[\lambda / \mathrm{m}]^{2.55} \mathrm{~K}$ and $T_{\mathrm{rcvr}}=0.1 T_{\mathrm{sky}}+40 \mathrm{~K}$. Simulations described in the document SKAl Imaging Science Performance $^{1}$ yield a noise level that should be within a factor of 2 of the natural instrument sensitivity.

\footnotetext{
${ }^{1}$ Braun, R., "SKA1 Imaging Science Performance", Document no. SKA-TEL-SKO-DD-XXX Revision A Draft 2
} 
The angular resolution corresponding to the $500 \mathrm{~m}$ radius core at $171 \mathrm{MHz}$ is $\theta_{\mathrm{b}}=7.3$ arc minutes, and the primary beam width for the $35 \mathrm{~m}$ station is $\Omega=3.5$ degrees (defined as centre to first null). For reference, the resulting rms noise in an image constructed in the manner described, for a frequency channel $\Delta v$ has the form

$$
\Delta T_{\mathrm{b}} \approx 0.05 \mathrm{mK}+0.66 \mathrm{mK}\left(\frac{1+z}{8.5}\right)^{2.55}\left(\frac{\Delta v}{1 \mathrm{MHz}} \frac{t_{\mathrm{int}}}{1000 \mathrm{hr}}\right)^{-1 / 2}\left(\frac{\theta_{\mathrm{b}}}{7^{\prime}}\right)^{-2} .
$$

Radio interferometers do not directly image the full range of spatial scales, but rather measure a frequency-dependent, complex visibility for each frequency channel and baseline $\mathbf{U}$ in their configuration. The measured visibility is a linear combination of signal and noise, with the latter proportional to the square-root of the effective fraction of the array that can observe a particular visibility $\mathbf{U}$, which is in turn proportional to the number density of baselines $n(\mathbf{U})$ that can observe the visibility (McQuinn et al., 2006). We simulate the thermal noise in a 3-dimensional visibilityfrequency cube (Geil et al., 2008), and then perform a 2-dimensional inverse Fourier transform in the $u v$-plane for each binned frequency in the bandwidth, which gives a realisation of the system noise in the image cube (i.e. sky coordinates). We scale the noise in each channel to have the variance described by equation (3.1). We construct the image using all baselines of up to $1000 \mathrm{~m}$, including both core stations and stations along the spiral arms.

\section{Simulated images of ionised structure with SKA1-LOW}

In Figure 3 we estimate the SKA1-LOW response to the ionisation structure in the GiggleZ $1 \mathrm{Gpc} / h$ cubed simulation in which galaxy formation is assumed to include an efficient SNe feedback. The top-left panel shows the model slice of depth $2 \mathrm{Mpc} / h$, which corresponds to $171 \mathrm{kHz}$ along the line-of-sight, at a central frequency of $175 \mathrm{MHz}$ for $\mathrm{HI}$ at $z=7.27$. As in Figure 1, our model has a neutral fraction of 0.45 at this redshift. In addition to the properties of the image noise, the array configuration directly impacts the features of the image that can be measured. On scales where the density of baselines is low or zero, features in the image cannot be observed. This includes image power on both small and large scales. Finite visibility coverage, determined by the baseline distribution, therefore truncates the visibilities that make up the observed image. Thus, in addition to dictating the behaviour of noise, the array configuration determines which properties of the HII regions can be imaged. The upper-right panel of Figure 3 shows an image of the simulation slice including only power on those scales measured by baselines of up to $1000 \mathrm{~m}$. Here we have assumed an estimate of the primary beam gain using the Fourier transform of a filled circle aperture.

Foreground emission, from Galactic synchrotron and extra-galactic point sources, will provide the largest challenge to measurement of the signal from reionisation. In this discussion we assume that resolved sources can be successfully removed, which should be practical at the proposed sensitivity of SKA1-LOW (Liu et al., 2009). We estimate the effects of removing the diffuse Galactic foregrounds, which can lower the contrast of observed images (Geil et al., 2008) by modelling the foreground continuum using a 2nd-order polynomial in the logarithm of frequency which is appropriate in the absence instrumental polarisation leakage (Geil et al., 2008, 2011). We perform fits with this functional form along the line-of-sight within a $20 \mathrm{MHz}$ bandwidth for each spatial pixel 

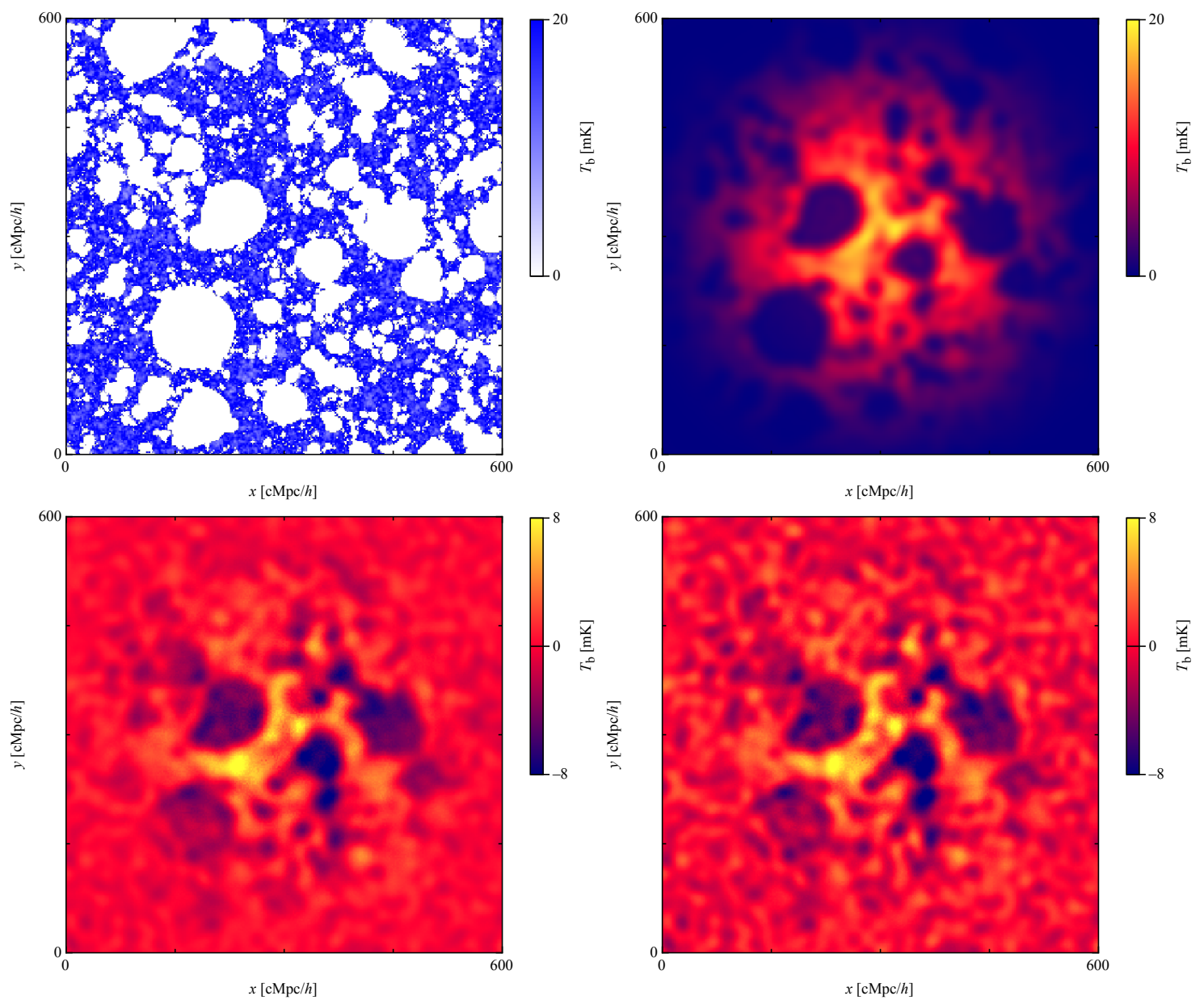

Figure 3: Simulations of the SKA1-LOW response to the ionisation structure in the Gigglez $1 \mathrm{Gpc} / \mathrm{h}$ simulation in which galaxy formation is assumed to include an efficient SNe feedback, and reionisation has progressed to a neutral fraction of 0.45 . The upper-left panel shows the model slice of depth $2 \mathrm{Mpc} / \mathrm{h}$ (corresponding to $171 \mathrm{kHz}$ at $z=7.27$ ). The upper-right panel shows an image of this slice assuming an estimate of the primary beam gain, accounting only for the power on scales that can be observed by baselines shorter than $1000 \mathrm{~m}$ (corresponding to the diameter of the core). The lower-left and lower-right panels show simulations of observed maps (without a primary beam correction) assuming the base-line SKA1-LOW with $1000 \mathrm{hr}$ integration and an early deployment where the collecting area is decreased by a factor of 2 . The model observations have a depth of $1.2 \mathrm{MHz}$, which corresponds to $14 \mathrm{Mpc} / \mathrm{h}$ along the line-of-sight, at a central frequency of $173 \mathrm{MHz}$ for $\mathrm{HI}$ at $z=7.27$, and is equivalent to the FWHP of the synthesised beam.

in the simulated image cube. We then subtract the best fit, leaving residual fluctuations around the foreground emission, which will include any residual continuum foregrounds, instrumental noise and the reionisation signal. The lower-left and lower-right panels of Figure 3 show simulated maps following diffuse foreground removal assuming $1000 \mathrm{hr}$ integrations with the base-line SKA1LOW design, and an early deployment of SKA1-LOW for which the sensitivity is decreased by a factor of 2 respectively. Here the slices are of depth $14 \mathrm{Mpc} / h$, which corresponds to $1.2 \mathrm{MHz}$ along the line-of-sight, and is equivalent to the full-width at half power (FWHP) of the synthesised 

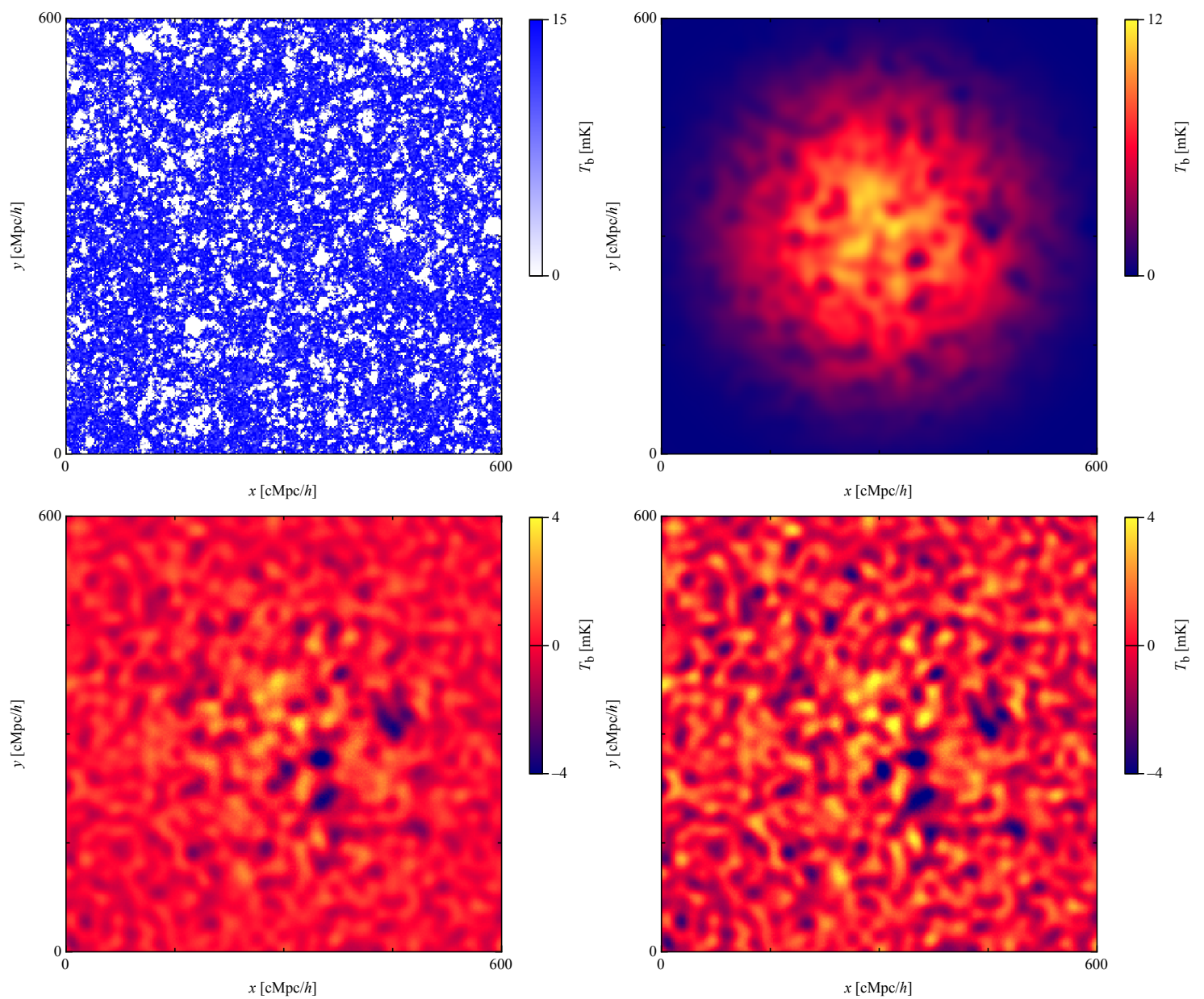

Figure 4: Simulations of the SKA1-LOW response to the ionisation structure in the GiggleZ $1 \mathrm{Gpc} / h$ simulation in which star formation is assumed to proceed in the absence of a strong SNe feedback. Details are as per Figure 3.

beam. The large HII regions produced by a model with $\mathrm{SNe}$ feedback can be imaged well by the SKA1-LOW baseline design, and marginally imaged with an early deployment of SKA1-LOW having half the sensitivity of the baseline design. The consequence of removing the foreground continuum, together with the fact that interferometers do not make zero-spacing measurements, results in a decreased contrast between ionised and non-ionised regions, and a loss of power from large-scale modes (Geil et al., 2008).

In Figure 4, we show examples of simulated maps for the case where HII regions are produced by galaxies in which star formation is assumed to proceed in the absence of a strong SNe feedback. Panels in this figure correspond to those in Figure 3. We find that while the configuration of the SKA1-LOW baseline design can observe the largest of the smaller HII regions generated by a galaxy formation model without $\mathrm{SNe}$ feedback, these will in practice be difficult to observe owing to noise and the effects of foreground subtraction which lower the contrast of the observed HII regions. The SKA1-LOW baseline design appears to be the minimum configuration necessary 

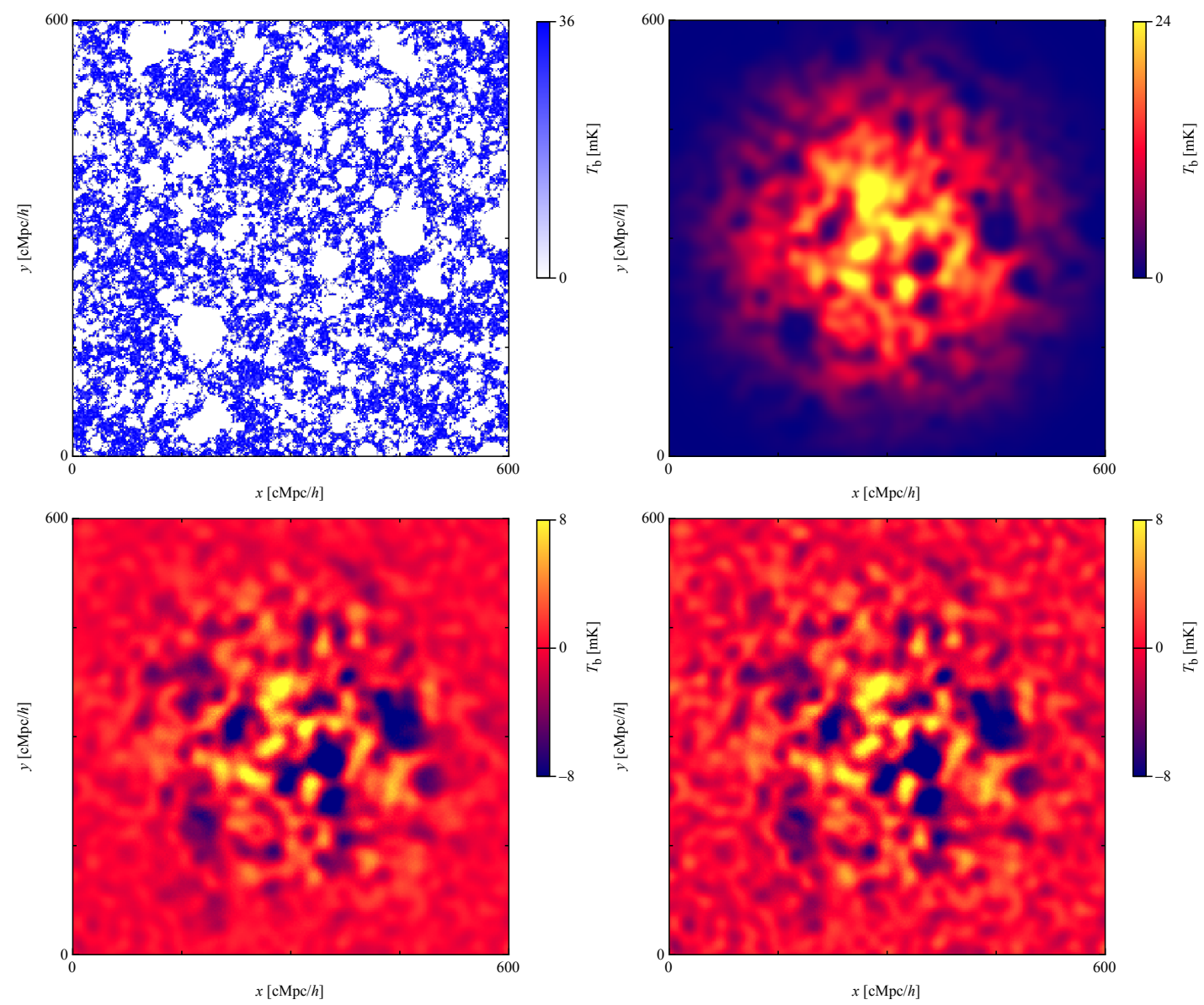

Figure 5: As per Figure 4, but assuming a more advanced stage of reionisation with a neutral fraction of 0.25 rather than 0.45 .

to image HII regions generated by this second galaxy formation model during the early-to-mid portions of the reionisation era. Figure 5 shows the corresponding maps in the scenario of galaxy formation without strong SNe feedback, where reionisation is more advanced with a smaller neutral fraction of 0.25 . The larger HII regions generated later in reionisation could be resolved by SKA1LOW. Note that although the HII regions in Figure 5 are of similar size to the case of galaxy formation with SNe feedback at an earlier phase of reionisation (Figure 3), the sensitivity of SKA1LOW would be sufficient to detect the excess of small-scale fluctuations seen in Figure 5, and would therefore provide a discriminant between these models.

The left and right panels of Figure 6 show simulated maps following diffuse foreground removal assuming $1000 \mathrm{hr}$ integrations with an SKA for which the sensitivity is increased by a factor of 4 over the SKA1-LOW baseline design. This sensitivity is achieved by increasing the density of stations in the spiral arms and size of the core (since increasing the density of the core would lead to a station filling factor greater than unity). As a result of the reduced FWHP of the synthesised beam, the model slice has a smaller depth of $7.4 \mathrm{Mpc} / h$, which corresponds to $0.45 \mathrm{MHz}$ along the line-of-sight (at a central frequency of $172 \mathrm{MHz}$ ). We find that the greater sensitivity and resolution 

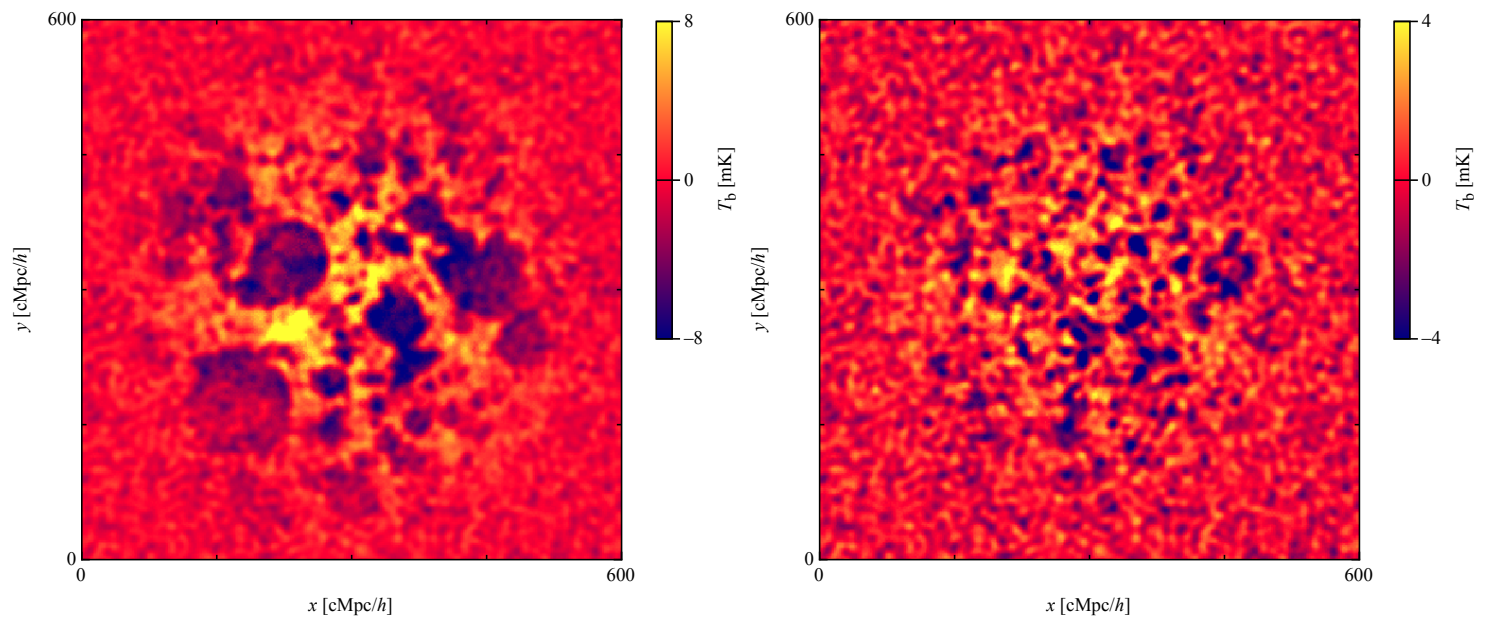

Figure 6: Simulations of the SKA response to the ionisation structure (without a primary beam correction) in the GiggleZ $1 \mathrm{Gpc} / h$ simulation in which star formation is assumed to include an efficient SNe feedback (left) and to proceed in the absence of a strong SNe feedback (right). In both cases reionisation is assumed to have progressed to a neutral fraction of 0.45 . For SKA, the sensitivity is assumed to increase by a factor of 4 (increasing the radius of the core by a factor of 2, and the density of stations in the arms by a factor of 4). The model observation has a depth of $7 \mathrm{Mpc} / h$, which corresponds to $0.6 \mathrm{MHz}$ along the line-of-sight (at a central frequency of $172 \mathrm{MHz}$ ).

of an SKA would allow more detailed imaging of HII regions generated by both the cases of galaxy formation with and without strong $\mathrm{SNe}$ feedback.

\section{Sharpness of HII regions}

Quasars have a harder spectrum than star forming galaxies, and this leads to thicker ionising fronts than is the case for a starburst driven HII region (Zaroubi \& Silk, 2005; Kramer \& Haiman, 2008). With sufficient angular resolution the contribution of hard ionising sources, such as miniquasars to reionisation could therefore be inferred from the structure of ionising fronts at the edge of HII regions (Tozzi et al., 2000). Similarly, the distribution of ionising sources surrounding a massive galaxy at the centre of an HII region prevents the boundaries of such HII regions from being sharp when viewed at finite resolution (Wyithe \& Loeb, 2007a). Rather, the clustering of sources near massive galaxies results in a spatially averaged neutral fraction that rises gradually towards large radii from an interior value near zero. As a result, a neutral hydrogen fraction corresponding to the global background value is typically reached only at a distance of 2-5 times the radius of the HII region around the central massive galaxy. This will lead to HII regions that look to have smooth edges unless observed at very high resolution. While detailed simulations remain to be done, inspection of Figures 3-6 implies that this science will require the sensitivity and resolution of SKA. 


\section{Field of view for SKA1-LOW}

Direct imaging of the ionisation structure on small scales during reionisation is challenging because of reduced sensitivity at high resolution. However simulations suggest that very large structures of $\mathrm{HI}$, with sizes of up to $100 \mathrm{Mpc}$ will still be present during the later stages of reionisation (Zaroubi et al., 2012). Such large areas of patchy reionisation in the IGM result from the clustering of the large-scale structure on scales of up to $\sim 120 h^{-1} \mathrm{Mpc}$, or $\sim 1$ degree (see Figure 1). Detection of these large-scale features may be possible at moderate significance with first generation arrays including LOFAR, and will be valuable for answering many cosmological questions (Zaroubi et al., 2012). However these expected large-scale features imply that the field of view for reionisation experiments performed with SKA1-LOW should have a size of at least several degrees.

At these very large scales, the light travel time can become comparable to the Hubble time, implying that light-cone effects become important. Thus, while HII regions can become arbitrarily large in a simulation at fixed proper time (as may be seen in Figure 1) during the brief period of overlap at the end of reionisation, the combined constraints of cosmic variance and light travel time imply a maximum observed HII region size at the end of the overlap epoch (as may be seen in Figure 2). This maximum size is found to have a value of $\sim 100 \mathrm{Mpc}$ (Wyithe \& Loeb, 2004a). In agreement with the simulations of Zaroubi et al. (2012), this implies that reionisation experiments with SKA1-LOW should be sensitive to a characteristic angular scale of $\sim 1$ degree for detection of the largest-scale $21 \mathrm{~cm}$ flux fluctuations near the end of reionisation, and have a field of view sufficiently large to image these features.

\section{Summary}

The properties of the ionisation structure of the IGM are sensitive to the unknown galaxy formation physics that prevailed during reionisation. This ionisation structure introduces nonGaussian statistics into the redshifted $21 \mathrm{~cm}$ fluctuation amplitudes which can only be studied through tomographic imaging, and will clearly discriminate between different galaxy formation scenarios. Imaging the ionisation structure of the IGM during reionisation is therefore a key goal for the SKA. As an example, we have shown that the SKA1-LOW baseline design with a $1 \mathrm{~km}$ diameter core would resolve HII regions expected from galaxy formation models that include strong feedback on low-mass galaxy formation. However detailed imaging of the smaller HII regions that result from galaxy formation in the absence of SNe feedback may require the greater sensitivity of SKA, particularly in the early-to-mid phases of reionisation. In addition to having baselines long enough to resolve the typical HII regions, the field of view for SKA1-LOW reionisation experiments should be at least several degrees across in order to image the largest HI structures towards the end of reionisation. The baseline design with $35 \mathrm{~m}$ diameter stations has a field of view within a single primary pointing which is marginally sufficient for this purpose.

\section{References}

Barkana, R. 2009, MNRAS, 397, 1454 
Bolton, J. S. \& Haehnelt, M. G. 2007, MNRAS, 381, L35

Bolton, J. S., Haehnelt, M. G., Warren, S. J., Hewett, P. C., Mortlock, D. J., Venemans, B. P., McMahon, R. G., \& Simpson, C. 2011, MNRAS, 416, L70

Bond, J. R. \& Myers, S. T. 1996, ApJS, 103, 1

Cen, R. \& Haiman, Z. 2000, ApJL, 542, L75

Furlanetto, S. R. \& Lidz, A. 2007, ApJ, 660, 1030

Furlanetto, S. R., Zaldarriaga, M., \& Hernquist, L. 2004, ApJ, 613, 16

Geil, P. M., Gaensler, B. M., \& Wyithe, J. S. B. 2011, MNRAS, 418, 516

Geil, P. M., Wyithe, J. S. B., Petrovic, N., \& Oh, S. P. 2008, MNRAS, 390, 1496

Harker, G. et. al.. 2009a, MNRAS, 397, 1138

Harker, G. J. A. et. al.. 2009b, MNRAS, 393, 1449

Iliev, I. T., Mellema, G., Ahn, K., Shapiro, P. R., Mao, Y., \& Pen, U.-L. 2014, MNRAS, 439, 725

Iliev et al. 2015, EoR modeling and simulations, in proceedings of "Advancing Astrophysics with the Square Kilometre Array", PoS(AASKA14)007

Kim, H.-S., Wyithe, J. S. B., Raskutti, S., Lacey, C. G., \& Helly, J. C. 2013, MNRAS, 428, 2467

Kohler, K., Gnedin, N. Y., Miralda-Escudé, J., \& Shaver, P. A. 2005, ApJ, 633, 552

Kramer, R. H. \& Haiman, Z. 2008, MNRAS, 385, 1561

Lagos, C. d. P., Bayet, E., Baugh, C. M., Lacey, C. G., Bell, T. A., Fanidakis, N., \& Geach, J. E. 2012, MNRAS, 426, 2142

Lidz, A., McQuinn, M., Zaldarriaga, M., Hernquist, L., \& Dutta, S. 2007, ApJ, 670, 39

Lidz, A., Zahn, O., Furlanetto, S. R., McQuinn, M., Hernquist, L., \& Zaldarriaga, M. 2009, ApJ, 690,252

Liu, A., Tegmark, M., \& Zaldarriaga, M. 2009, MNRAS, 394, 1575

McQuinn, M., Zahn, O., Zaldarriaga, M., Hernquist, L., \& Furlanetto, S. R. 2006, ApJ, 653, 815

Mellema, G., Iliev, I. T., Pen, U.-L., \& Shapiro, P. R. 2006, MNRAS, 372, 679

Mellema et al. 2015, in proceedings of "Advancing Astrophysics with the Square Kilometre Array", PoS(AASKA14)010

Mesinger, A. \& Furlanetto, S. 2007, ApJ, 669, 663

Park, J., Kim, H.-S., Wyithe, J. S. B., \& Lacey, C. G. 2014, MNRAS, 438, 2474

Poole, G. B. et. al.. 2014, ArXiv e-prints 1407.0390

Thomas, R. M. et. al.. 2009, MNRAS, 393, 32

Tozzi, P., Madau, P., Meiksin, A., \& Rees, M. J. 2000, ApJ, 528, 597

Valdés, M., Ciardi, B., Ferrara, A., Johnston-Hollitt, M., \& Röttgering, H. 2006, MNRAS, 369, L66

Wyithe, J. S. B. \& Loeb, A. 2004a, Nature, 427, 815

-. 2004b, ApJ, 610, 117

-. 2007a, MNRAS, 374, 960

-. 2007b, MNRAS, 375, 1034

Wyithe, J. S. B. \& Morales, M. F. 2007, MNRAS, 379, 1647

Zahn, O., Lidz, A., McQuinn, M., Dutta, S., Hernquist, L., Zaldarriaga, M., \& Furlanetto, S. R. 2007, ApJ, 654, 12

Zaroubi, S. et. al.. 2012, MNRAS, 425, 2964

Zaroubi, S. \& Silk, J. 2005, MNRAS, 360, L64 